\title{
Dilution testing using rapid diagnostic tests in a HIV diagnostic algorithm: a novel alternative for confirmation testing in resource limited settings
}

\author{
Leslie Shanks ${ }^{1 *}$, M. Ruby Siddiqui ${ }^{2}$, Almaz Abebe $^{3}$, Erwan Piriou${ }^{1}$, Neil Pearce $^{4}$, Cono Ariti $^{4}$, Johnson Masiga ${ }^{1}$, \\ Libsework Muluneh $^{3}$, Joseph Wazome ${ }^{1}$, Koert Ritmeijer ${ }^{1}$ and Derryck Klarkowski ${ }^{1}$
}

\begin{abstract}
Background: Current WHO testing guidelines for resource limited settings diagnose HIV on the basis of screening tests without a confirmation test due to cost constraints. This leads to a potential risk of false positive HIV diagnosis. In this paper, we evaluate the dilution test, a novel method for confirmation testing, which is simple, rapid, and low cost. The principle of the dilution test is to alter the sensitivity of a rapid diagnostic test (RDT) by dilution of the sample, in order to screen out the cross reacting antibodies responsible for falsely positive RDT results.
\end{abstract}

Methods: Participants were recruited from two testing centres in Ethiopia where a tiebreaker algorithm using 3 different RDTs in series is used to diagnose HIV. All samples positive on the initial screening RDT and every 10th negative sample underwent testing with the gold standard and dilution test. Dilution testing was performed using Determine $^{\mathrm{TM}}$ rapid diagnostic test at 6 different dilutions. Results were compared to the gold standard of Western Blot; where Western Blot was indeterminate, PCR testing determined the final result.

Results: 2895 samples were recruited to the study. 247 were positive for a prevalence of $8.5 \%$ (247/2895). A total of 495 samples underwent dilution testing. The RDT diagnostic algorithm misclassified 18 samples as positive. Dilution at the level of 1/160 was able to correctly identify all these 18 false positives, but at a cost of a single false negative result (sensitivity $99.6 \%, 95 \%$ Cl 97.8-100; specificity $100 \%, 95 \%$ Cl: 98.5-100). Concordance between the gold standard and the 1/160 dilution strength was $99.8 \%$.

Conclusion: This study provides proof of concept for a new, low cost method of confirming HIV diagnosis in resource-limited settings. It has potential for use as a supplementary test in a confirmatory algorithm, whereby double positive RDT results undergo dilution testing, with positive results confirming HIV infection. Negative results require nucleic acid testing to rule out false negative results due to seroconversion or misclassification by the lower sensitivity dilution test. Further research is needed to determine if these results can be replicated in other settings.

Trial registration: ClinicalTrials.gov, NCT01716299.

\section{Background}

The diagnosis of HIV is made on the basis of a reactive screening test or tests followed by a confirmation test. However due to issues of cost, the WHO currently recommends that confirmation testing is not performed in resource limited settings, and instead that diagnosis be made on the basis of an algorithm employing 2-3 rapid diagnostic tests (RDTs) [1]. This strategy has allowed

\footnotetext{
* Correspondence: Leslie.shanks@amsterdam.msf.org

'Médecins Sans Frontières, Amsterdam, The Netherlands

Full list of author information is available at the end of the article
}

life-saving scale up of HIV diagnosis, as it permits testing to be decentralized outside of the laboratory. The compromise is that without a confirmation test, some individuals will be falsely diagnosed as HIV positive. This risk of false positive HIV diagnosis on the basis of 2 RDT positive results has been shown in a number of settings with different RDTs [2-5]. The risk is increased in lower prevalence populations. The mechanism causing false positive reactions on serological tests is that of non-HIV antibodies cross-reacting with the test antigens [6]. Given the consequences for individuals in

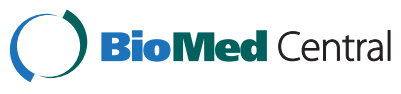

(C) 2015 Shanks et al.; licensee BioMed Central. This is an Open Access article distributed under the terms of the Creative Commons Attribution License (http://creativecommons.org/licenses/by/4.0), which permits unrestricted use, distribution, and reproduction in any medium, provided the original work is properly credited. The Creative Commons Public Domain Dedication waiver (http://creativecommons.org/publicdomain/zero/1.0/) applies to the data made available in this article, unless otherwise stated. 
terms of the psychological impact, effect on family and community, and possible health consequences of unnecessary exposure to antiretroviral drugs, our group has called for implementation of routine confirmation testing [3]. However, the gold standard for confirmation of HIV testing has been Western Blot (WB) or indirect immunofluorescence assay (IFA) neither of which is suitable for use in peripheral laboratories. Traditional confirmation tests also have limitations in identifying recent seroconversion, can give indeterminate results, and do not allow discrimination between HIV 1 \& 2 infections. These limitations have led the US to introduce new guidelines that employ a supplementary testing algorithm rather than a single confirmation test $[7,8]$. Samples repeatedly positive on screening assays, are given a supplementary test to confirm infection, and if negative go on to nucleic acid testing (NAT) to rule out a false negative result due to early seroconversion. The only supplementary test currently approved by the FDA is Bio-Rad Multispot HIV-1/HIV-2 Rapid Test. It is a single use flow-through rapid test that yields a result in $15 \mathrm{~min}$., and is able to discriminate between HIV $1 \& 2$. Bio-Rad Geenius HIV 1/2 Confirmatory Assay, is another single use rapid test that is being evaluated as a supplementary test but is not yet approved for this use by the FDA. We are not aware of any published evaluations done outside of reference laboratories of either test and current pricing limits their use in resourcelimited settings. We use a low cost confirmation test which we have shown is feasible for use in a peripheral laboratory, the Orgenics Immunocomb ${ }^{\bullet}$ II, HIV $1 \& 2$ Combfirm or OIC. [3]. However this test is not CE marked nor has it been evaluated by WHO. There is therefore an urgent need to develop new confirmation tests or methodologies that are low cost and feasible for use in resource-limited settings.

In this paper, we describe a new methodology to confirm positive HIV screening tests, using serial sample dilution of RDTs. We postulate that by decreasing the sensitivity of the HIV RDT through serially diluting the sample, it will be possible to screen out false positive samples, and correctly identify true positives.

The work reported here formed part of a larger study on HIV diagnostics [9]. In Ethiopia, a tiebreaker regimen consisting of 3 RDTs in series is the national algorithm. HIV $(1+2)$ Antibody Colloidal Gold (KHB, Shanghai Kehua Bio-engineering Co Ltd, China) is used as a screening test, followed by HIV 1/2 STAT-PAK (Chembio Diagnostics, USA) if positive. Where the result of STAT$\mathrm{PAK}^{\circ}$ is discordant with $\mathrm{KHB}$, a third test, Unigold HIV (Trinity Biotech, Ireland), is used as a tiebreaker to determine the result. Médecins Sans Frontières-Holland (MSF), in response to reported cases of false positive results [10], uses an alternate algorithm in Ethiopia, consisting of two
RDTs (KHB, STAT-PAK ${ }^{\circ}$ ) in series, followed by the OIC confirmation test. The primary objective of the study was to evaluate the performance of these two algorithms. Secondary objectives, in addition to evaluating dilution testing, were to evaluate the positive predictive value of weakly reactive RDT test lines [9] and to determine if visceral leishmaniasis (VL), a disease endemic in the region, was associated with an increased risk of false positive reactions. In this paper we focus on the dilution testing objective.

\section{Methodology \\ Setting}

The study was conducted in 2 sites in north-western Ethiopia: a MSF supported health centre in Abdurafi, Amhara Region and a hospital in Humera, Tigray Region. The populations included residents as well as high numbers of migrant workers who were present seasonally.

\section{Inclusion criteria}

All clients, aged $>=5$ years, presenting to be tested for HIV in the study sites were offered participation in the study. All study participants underwent informed consent procedures and had a written consent form signed by the participant or the guardian.

\section{Sample size}

A sample size of $200 \mathrm{KHB}$ positive and $200 \mathrm{KHB}$ negative participants was chosen based on the WHO guidance for evaluation of RDTs [11]. To achieve the sample, all KHB positive samples were included along with every 10th KHB negative sample until a minimum of 200 positive samples was reached. In order to increase power for the secondary objective on VL, additional participants were recruited until a total of 90 individuals with VL were included. Dilution testing was performed on the total sample set.

\section{Testing}

Initial testing was done at the Counselling and Testing (CT) centres. Laboratory technicians, blinded to the CT results, re- tested each sample on plasma with the 3 RDTs in the national algorithm (KHB, STAT-PAK ${ }^{\circ}$ and Unigold $\left.^{\mathrm{Tm}}\right)$. Tests were performed according to manufacturer's instructions and invalid tests discarded and repeated on new test devices. All samples underwent testing by Western Blot (WB) with technicians blinded to earlier results using MP Diagnostics HIV Blot 2.2. Interpretation of results was based on American Red Cross recommendations [12].

Samples indeterminate on WB were repeated and if still indeterminate, underwent DNA PCR examination using Roche Amplicor DNA v1.5 on dry blood spots (DBS) at the Ethiopian Health and Nutrition Research Institute (EHNRI) laboratory based in Addis Ababa. Global Clinical and Viral Laboratory in South Africa 
provided quality control for PCR by repeating the samples to confirm results using the Cobas AmpliPrep/ Cobas TaqMan HIV-1 Qual test. Samples were sent as dry blood spots (DBS) by international courier, packaged in accordance with IATA regulations for such samples. Where results between the two labs were discrepant, the result from South Africa was used.

The final gold standard result was that of Western Blot, and where Western Blot was indeterminate, the PCR result.

\section{Dilution technique}

Patient plasma was diluted with seronegative plasma from healthy blood donors using a micropipette and microtitration plate. Plasma was defined as seronegative when negative on 2 RDTs and on WB. $10 \mu \mathrm{L}$ of patient plasma was first diluted 1:10 in $90 \mu \mathrm{L}$ of negative plasma. This was followed by a serial 4-fold dilution from 1:10 to 1:10,240 in negative plasma.

Testing was performed on Determine ${ }^{\text {Tx }}$ HIV-1/2 (Alere Laboratories, Japan) using $50 \mathrm{~L}$ of diluted sample and read within 15 minutes following manufacturer's instructions. Tests were interpreted as positive if there was any colouration of the test line. The highest dilution that gave a positive result was recorded. Where the lowest dilution $(1 / 10)$ was negative, the sample was reported as negative. Invalid tests, where the control line did not appear, were discarded and repeated on a new test device according to manufacturers' instructions.

\section{Quality control}

All staff underwent training on the study standard operating procedures by the MSF laboratory supervisor, and received regular monitoring and supervision.

\section{Analysis}

Predictive values and sensitivity and specificity were estimated from the $2 \times 2$ table of observed results after weighting based on the sampling proportion of the KHB positive and negative samples. Confidence intervals for each of the test parameters were calculated using exact binomial intervals.

The proportion of total specimens with the same classification measured concordance between the gold standard and experimental methodology. The kappa statistic was calculated to assess agreement. Statistical analysis was done using Stata version 12 (StataCorp, College Station, Texas, USA).

\section{Ethical review}

The study received approval from the MSF Ethics Review Board, the Ethiopian Health and Nutrition Research Institute Research and Ethical Clearance Committee, and the National Research Ethics Review Committee, Ministry of Science and Technology in Ethiopia.

\section{Results}

2897 individuals were recruited to the study. One sample was excluded due to missing WB/PCR results, and a duplicate was dropped, giving a total of 2895 in the final analysis. 495 were selected for testing by dilution and with the gold standard. 247 were positive on the gold standard for a prevalence of $8.5 \%(247 / 2895)$.

The serial KHB/STAT-PAK ${ }^{\circ}$ algorithm correctly identified 247 positives, yielded one false positive and no false negatives for a sensitivity of $100 \%$ (95 \% CI: 98.5-100), specificity of $100 \%$ (95\% CI: 99.8-100), negative predictive value (NPV) of $100 \%$ (95\% CI: 98.5-100) and positive predictive value (PPV) of $99.6 \%$ (95\% CI: 97.8-100).

The tiebreaker KHB/STAT-PAK ${ }^{\circ} /$ Unigold $^{\text {tax }}$ algorithm correctly identified 247 positives, yielded 18 false positives, and no false negatives for a sensitivity of $100 \%$ (95 \% CI: 98.5-100), specificity of 99.3 \% (95 \% CI: 98.999.6), NPV of $100 \%$ (95\% CI: 98.4-100) and PPV of 93.2 \% (95 \% CI: 89.5-95.9).

\section{Results of dilution testing}

Results for the performance of each dilution can be found in Tables $1-2$. The $1 / 40$ dilution correctly identified 247 positives and 246 negatives, and had two false positives. The $1 / 160$ dilution correctly identified 246 positives and 248 negatives with no false positives, one false negative, while the $1 / 640$ dilution had no false positives and 3 false negatives.

The sensitivity of the 1/160 dilution was $99.6 \%$ (95 \% CI: 97.8-100) with a NPV of $100 \%$ (95 \% CI: 99.8-100) and a specificity and PPV of $100 \%$ (95\% CI: 98.5-100).

Concordance between the dilution test and the gold standard of WB resolved by PCR is shown in Table 1 . Concordance of the best performing dilution, 1/160, with WB alone was $87.15 \%(\mathrm{kappa}=0.771)$. Excluding the $64 \mathrm{WB}$ indeterminate results gave $100 \%$ concordance (kappa $=1.0)$.

Incorporating the dilution tests into the tiebreaker algorithm improved the specificity and PPV at the lower dilutions of $1 / 10$ and $1 / 40$. Complete results are shown in Tables 3-4.

\section{Results on discordant RDTs}

The serial algorithm KHB/STAT-PAK ${ }^{\bullet}$ had 24 discordant results, all of which were resolved negative. The 1/40 strength misidentified one discordant as positive whereas the 1/10 dilution had 3 false positives. The higher dilution strengths from $1 / 160$ and up were able to correctly identify all 24 as negative. An alternate serial $\mathrm{KHB} /$ Unigold $^{\mathrm{Tw}}$ algorithm had 9 discordant results, 2 of which were positive on gold standard. All dilution strengths correctly identified all 9 discordants. 
Table 1 Results of the dilution test compared to gold standard $(\mathrm{N}=495)$

\begin{tabular}{|c|c|c|c|c|c|c|}
\hline \multirow[t]{2}{*}{ Dilution } & \multirow[t]{2}{*}{ Result } & \multirow[t]{2}{*}{ Number } & \multicolumn{2}{|c|}{ Number with gold standard result } & \multirow[t]{2}{*}{ Concordance } & \multirow[t]{2}{*}{ Kappa value* } \\
\hline & & & Positive (\%) & Negative (\%) & & \\
\hline \multirow[t]{2}{*}{$1 / 10$} & Positive & 259 & $247(95.3)$ & $12(4.6)$ & 97.6 & 0.952 \\
\hline & Negative & 236 & 0 & $236(100)$ & & \\
\hline \multirow[t]{2}{*}{$1 / 40$} & Positive & 249 & $247(99.2)$ & $2(0.8)$ & 99.6 & 0.992 \\
\hline & Negative & 246 & 0 & $246(100)$ & & \\
\hline \multirow[t]{2}{*}{$1 / 160$} & Positive & 246 & $246(100)$ & 0 & 99.8 & 0.996 \\
\hline & Negative & 249 & $1(0.4)$ & $248(99.6)$ & & \\
\hline \multirow[t]{2}{*}{$1 / 640$} & Positive & 244 & $244(100)$ & 0 & 99.4 & 0.988 \\
\hline & Negative & 251 & $3(1.2)$ & $248(98.8)$ & & \\
\hline \multirow[t]{2}{*}{$1 / 2560$} & Positive & 240 & $240(100)$ & 0 & 98.6 & 0.972 \\
\hline & Negative & 255 & $7(2.8)$ & $248(97.2)$ & & \\
\hline \multirow[t]{2}{*}{$1 / 10,240$} & Positive & 221 & $221(100)$ & 0 & 94.8 & 0.895 \\
\hline & Negative & 274 & $26(9.5)$ & $248(90.5)$ & & \\
\hline TOTAL & & 495 & 247 (49.9) & $248(50.1)$ & & \\
\hline
\end{tabular}

*All $\mathrm{p}$ values for the kappa statistics are $<0.001$

\section{Discussion}

The dilution test was able to correctly identify all 18 false positive results at a level of $1 / 160$ with a specificity of $100 \%$. However the improved specificity came at a cost of sensitivity, as the sensitivity worsened with increasing dilutions.

To further understand the sensitivity of the new methodology, we examined the false negative results at lower dilutions to determine if they were more likely to be due to early seroconversion or to poor sensitivity of the dilution test. One gold standard positive sample tested positive at a dilution of $1 / 40$ but negative at $1 / 160$. This sample had a weakly positive test line on all 3 RDTs, an indeterminate result on WB (+gp160, +p24) and a positive result on PCR. This individual was tested due to presence of a sexually transmitted infection and listed the most recent exposure as 19 days prior to testing. CD4 was 428 . This sample was suggestive of seroconversion. There were 2 additional false negative results at the dilution level of $1 / 640$. One was positive on KHB and Unigold $^{\mathrm{m}}{ }^{\mathrm{w}}$, weakly reactive for STAT-PAK ${ }^{\circ}$, and positive on WB. Reason for testing was risk of exposure with most recent exposure 11 days prior to testing. CD4 was 260. The second sample was positive on all 3 RDTs, WB and PCR. Reason for testing was pre-marriage screening and no exposure risk was listed. CD4 was 647. Both of these samples were unlikely to be seroconversion. More data is needed, however this suggests that occasional true positive samples with established HIV infection will be misclassified by dilution. This is relevant, as instructing the patient to return to be re-tested after several weeks as is done now when early seroconversion is suspected, may not correctly resolve these false negatives.

Given the risk of false negative results with the dilution test, we conclude that the dilution test cannot replace WB as a confirmation test. However its ability to differentiate false positives from true positives, suggests that it has potential to be used as a supplementary test in a confirmatory algorithm where RDT algorithm positive samples are confirmed with dilution, and negative dilution samples are referred to a higher-level laboratory for NAT. The referral for NAT is facilitated by the recently increased availability of PCR testing on dried blood spots for infant testing.

\section{Comparison with existing supplementary tests}

The performance of dilution as a supplementary test compares well to published evaluations of both Multi-

Table 2 Performance characteristics of the dilution test compared to gold standard $(\mathrm{N}=2895)$

\begin{tabular}{lllll}
\hline Dilution & Sensitivity $(95 \% \mathrm{Cl})$ & Specificity $(95 \% \mathrm{Cl})$ & PPV $(95 \% \mathrm{Cl})$ & $\mathrm{NPV}(95 \% \mathrm{Cl})$ \\
\hline $1 / 10$ & $100 \%(98.5-100)$ & $96.3 \%(95.5-97.0)$ & $71.6 \%(66.5-76.3)$ & $99.6 \%(98.5-100)$ \\
$1 / 40$ & $100 \%(98.5-100)$ & $99.5 \%(99.2-99.7)$ & $95.1 \%(91.6-97.3)$ & $100 \%(98.5-100)$ \\
$1 / 160$ & $99.6 \%(97.8-100)$ & $100 \%(98.5-100)$ & $100 \%(98.5-100)$ & $100 \%(99.8-100)$ \\
$1 / 640$ & $98.8 \%(96.5-99.8)$ & $100 \%(98.5-100)$ & $100 \%(98.5-100)$ & $99.9 \%(99.7-100)$ \\
$1 / 2560$ & $97.2 \%(94.3-98.9)$ & $100 \%(98.5-100)$ & $100 \%(98.5-100)$ & $99.7 \%(99.5-99.9)$ \\
$1 / 10,240$ & $89.5 \%(85.0-93.0)$ & $100 \%(98.5-100)$ & $100 \%(98.3-100)$ & $99.0 \%(98.6-99.4)$ \\
\hline
\end{tabular}


Table 3 Results of KHB/STAT-PAK ${ }^{\oplus} /$ Unigold ${ }^{\mathrm{TM}}$ tiebreaker algorithm with dilution as a supplementary test compared to gold standard $(\mathrm{N}=495)$

\begin{tabular}{|c|c|c|c|c|c|c|}
\hline \multirow[t]{2}{*}{ Algorithm } & \multirow[t]{2}{*}{ Result } & \multirow[t]{2}{*}{ Number } & \multicolumn{2}{|c|}{ Number with gold standard result } & \multirow[t]{2}{*}{ Concordance } & \multirow[t]{2}{*}{ Kappa value } \\
\hline & & & Positive (\%) & Negative (\%) & & \\
\hline \multirow[t]{2}{*}{$1 / 10$} & Positive & 251 & $247(98.4)$ & $4(1.6)$ & $99.2 \%$ & 0.984 \\
\hline & Negative & 244 & 0 & $244(100)$ & & \\
\hline \multirow[t]{2}{*}{$1 / 40$} & Positive & 248 & $247(99.6)$ & $1(0.4)$ & $99.8 \%$ & 0.996 \\
\hline & Negative & 247 & 0 & $247(100)$ & & \\
\hline \multirow[t]{2}{*}{$1 / 160$} & Positive & 246 & $246(100)$ & 0 & $99.8 \%$ & 0.996 \\
\hline & Negative & 249 & $1(0.4)$ & $248(99.6)$ & & \\
\hline \multirow[t]{2}{*}{$1 / 640$} & Positive & 244 & $244(100)$ & 0 & $99.4 \%$ & 0.988 \\
\hline & Negative & 251 & $3(1.2)$ & $248(98.8)$ & & \\
\hline \multirow[t]{2}{*}{$1 / 2560$} & Positive & 240 & $240(100)$ & 0 & $98.6 \%$ & 0.972 \\
\hline & Negative & 255 & $7(2.8)$ & $248(97.3)$ & & \\
\hline \multirow[t]{2}{*}{$1 / 10,240$} & Positive & 221 & $221(100)$ & 0 & $94.8 \%$ & 0.895 \\
\hline & Negative & 274 & $26(9.5)$ & $248(90.5)$ & & \\
\hline TOTAL & & 495 & 247 (49.9) & $248(50.1)$ & & \\
\hline
\end{tabular}

*All $p$ values for the kappa statistics are $<0.001$

spot and Geenius. An American study of Multispot in an algorithm with Architect $4^{\text {th }}$ generation immunoassay showed that Multispot identified a similar proportion of true positives as Western Blot, $93.7 \%$ versus $94.4 \%$ [13]. There were $90(13.8 \%)$ samples that were positive on the screening assay and negative on Multispot. These were resolved by NAT, to reveal $47.8 \%$ were false positives on the screening assay and $52.2 \%$ were early seroconversion. A Canadian study compared Multispot with Geenius on a panel of samples [14]. Sensitivity for both was $100 \%$, however specificity was $99.1 \%$ for Multispot and $96.3 \%$ for Geenius $(\mathrm{p}=0.688)$. Kappa value for agreement between the two tests was 0.96 (0.92-0.99).

An ideal confirmation test for resource limited settings would be low cost, simple and rapid, robust in terms of temperature and environmental conditions, with both high sensitivity and specificity and few indeterminate results. The dilution test uses RDTs that are commonly available in sites doing HIV testing. It requires less skill than the WB, and is similar in skill level to that of the $\mathrm{OIC}$ as it also requires precise pipetting techniques. In most cases, only 2 dilutions need to be tested, giving a cost of approximately $\$ 3$ USD and a turnaround time of 30-40 minutes. This compares to 2 hours for the OIC, 15 minutes for the Multi-spot and 3 hours for WB. An advantage is that there are no indeterminate results, as with OIC, Multi-spot and WB. Indeterminate results are undesirable, as they require individuals to come back for further testing with the attendant risk of failure to follow up. The dilution test can not be used to definitively rule out HIV infection due to the risk of false negatives and requires all negative results to be tested with NAT. However for the majority of screen positive samples, same day results would be available. In our cohort, using the tiebreaker algorithm presently in use in Ethiopia, this would mean 246 of 265 (92.8\%) screen positives could be immediately confirmed at a dilution of $1 / 160$, while 19 would go on to further testing to ultimately identify 18 false positives. In other words, one positive sample would be unnecessarily subjected to an additional test. A dilution of $1 / 640$ would result in 244/265 (92.1\%) positives immediately confirmed, and 21 sent for NAT.

Table 4 Performance characteristics of the KHB/STAT-PAK ${ }^{\circledast} /$ Unigold $^{\text {TM }}$ tiebreaker algorithm with dilution as a supplementary test compared to gold standard $(\mathrm{N}=2895)$

\begin{tabular}{lllll}
\hline Dilution & Sensitivity $(95 \% \mathrm{Cl})$ & Specificity $(95 \% \mathrm{Cl})$ & PPV $(95 \% \mathrm{Cl})$ & $\mathrm{NPV}(95 \% \mathrm{Cl})$ \\
\hline $1 / 10$ & $100 \%(95.8-100)$ & $99.9 \%(99.6-100)$ & $98.4 \%(96.0-99.6)$ & $100 \%(98.5-100)$ \\
$1 / 40$ & $100 \%(98.5-100)$ & $100 \%(99.8-100)$ & $99.6 \%(97.8-100)$ & $100 \%(98.5-100)$ \\
$1 / 160$ & $99.6 \%(97.8-100)$ & $100 \%(98.5-100)$ & $100 \%(98.5-100)$ & $100 \%(99.8-100)$ \\
$1 / 640$ & $98.8 \%(96.5-99.8)$ & $100 \%(98.5-100)$ & $100 \%(98.5-100)$ & $99.9 \%(99.7-100)$ \\
$1 / 2560$ & $97.2 \%(94.3-98.9)$ & $100 \%(98.5-100)$ & $100 \%(98.5-100)$ & $99.7 \%(99.5-99.9)$ \\
$1 / 10,240$ & $89.5 \%(85.0-93.0)$ & $100 \%(98.5-100)$ & $100 \%(98.3-100)$ & $99.0 \%(98.6-99.4)$ \\
\hline
\end{tabular}


The 2013 US diagnostic algorithm emphasizes the importance of detection of acute HIV infection, and discrimination between HIV 1 \& 2. Dilution testing used as part of a supplementary testing algorithm with NAT, would identify early seroconversion. Discrimination between HIV 1 \& 2 would not be addressed aside from that gleaned from discriminatory HIV RDTs. This is a similar limitation to Western Blot, which is known to be inaccurate at discriminating between HIV 1 \& 2 .

\section{Theoretical basis for dilution methodology}

Our methodology is based on the principles used to identify recent HIV infection for the purposes of incidence surveys. Here recent infection versus established infection is distinguished through an algorithm whereby samples are tested for HIV with enzyme immunoassays (EIA) with high sensitivity. Positive samples then go on to get a second, less sensitive test. The less sensitive test is an EIA test that is modified by use of a diluted sample and reduced incubation time. Samples positive on both tests are determined to be established infection, while those positive on the sensitive (S) test but negative on the less sensitive (LS) test are designated recent infection. This S/LS methodology is based on the principle that antibody titres increase over a period of several months after initial infection. Samples with low antibody titres test negative on EIA when the sample is diluted, whereas samples with higher titres are persistently positive [15]. Following on the work of Constantine [16], Soroka et al. proposed a variation for resource limited settings, whereby RDTs are substituted for the EIA in the S/LS algorithm [17]. Here dilution is again used to alter the sensitivity of the RDTs. A positive result on the first RDT screening test, followed by a negative RDT result on the diluted sample suggests recent infection. This algorithm has been successfully employed in incidence surveys using different RDTs and at differing dilution levels $[18,19]$.

Broad spectrum antibodies are produced in the early immune response to infectious disease antigens and can cause non-specific cross reactivity in serological testing [6]. These antibodies are expected to have low avidity, as has been demonstrated by work in blood donors [20]. In proposing dilution as a methodology to confirm HIV infection, we postulate that dilution will distinguish both the low avidity antibodies, as well as the possibly low titres [21] of the cross-reacting antibodies responsible for false reactivity. We found data to support this application of dilution testing in a report from Ghana using Soroka's methodology to identify incident HIV infection that describes how acute seroconversion was confused with false reactivity [22]. 76 samples repeatedly positive on Genescreen Ultra HIV Ag-Ab ELISA were tested on Determine $^{\mathrm{TM}}$ with the S/LS methodology. 41 were positive on the sensitive RDT, and of these 18 were positive on dilution, while 23 were negative on dilution. Confirmation with INNO-LIA ${ }^{\mathrm{TM}}$ revealed that all 35 Determine $^{\mathrm{TM}}$ nonreactive results were true negatives, and only 1 of 23 samples negative on dilution was HIV positive. All those positive on dilution were true positives, suggesting that dilution was able to distinguish the cross reactivity from true positivity.

\section{Choice of RDT}

We chose to use Determine ${ }^{\mathrm{TM}}$ as the RDT as it has high sensitivity and is in common use in many countries. We also had experience with piloting dilution testing on Determine $^{\mathrm{Tm}}$ in the Democratic Republic of Congo and therefore had programmatic data on its performance. We diluted samples with normal saline to $1 / 5000$ and used the OIC confirmation test as the gold standard. The proportion of agreement with OIC for 263 samples positive on both Determine ${ }^{\mathrm{TM}}$ and Unigold $^{\mathrm{TM}}$ was $89.2 \%$ (kappa 0.423) when indeterminates on OIC were excluded. The dilution test identified all 23 RDT algorithm false positives, and introduced 5 false negatives. A limitation is that no NAT was done, so it is possible that some of the false positives identified on gold standard were in fact early seroconversion.

\section{Use of dilution with RDT discordant samples}

Finally, an unexpected finding in our study was the ability of dilution testing at a level of $1 / 160$ to correctly resolve all 33 RDT discordant results from the serial algorithms in our study. Discordant results occur when one RDT is positive and the other negative on a serial or parallel algorithm. They are undesirable because they result in an inconclusive result for the client that requires re-testing in two weeks [1]. National programmes often use a tiebreaker algorithm to avoid these inconclusive results with their attendant risk of loss to follow up despite the known high risk of false positives results with the tiebreaker algorithm [2, 9, 23, 24]. Our results suggest that dilution testing has the potential to immediately confirm positive results and to allow a preliminary negative result to be given to clients with discordant RDT results. Discordant samples negative on dilution would still require follow up testing in two weeks and/or referral for NAT. Further formal study is needed to explore this potential role for dilution testing in resolving discordant RDT results.

\section{Limitations}

The strength of our study is that NAT testing was available to resolve indeterminate WB samples which made it possible to rule out early seroconversion as a potential cause of false positive results. It is limited by its application in a single cohort in Ethiopia with a single 
RDT. More work is needed to determine whether our results are replicable in other locations and with other RDTs. In addition, each site using dilution as a supplementary test would need to establish an appropriate dilution cut-off prior to using the test. The methodology would be similar to what has been used in incidence surveys [17].

\section{Conclusion}

Our study is a first step in providing proof of concept for a new, cost effective method of confirming HIV diagnosis in resource-limited settings. It has potential for use as a supplementary test in a confirmatory algorithm, whereby double positive RDT results are tested by dilution, with positive results confirming HIV infection. Negative dilution results would require NAT testing to rule out false negative results either due to seroconversion or misclassification by the lower sensitivity dilution test. Further research is needed to determine if our results can be replicated in other settings.

\section{Competing interests}

The authors declare that they have no competing interests.

\section{Authors' contributions}

LS: conceived of the study, analysed the data, and wrote the first draft of the paper. RS: coordinated the study, data collation, validation and analysis. AA: coordinated the study from EHNRI side and reviewed the draft paper. EP: Contributed to data analysis and interpretation, and writing of manuscript. NP, CA: provided the methodology for the analysis. CA performed the statistical analysis of the test performances. JM: Was involved in supervision of the lab, data entry and performing confirmatory testing. LM: coordinated and followed up the lab work from the EHNRI side and did supportive visits to the study sites. KR: contributed to the study conception, data analysis and interpretation, and critical revision of the draft manuscript. JW: Contributed to the study conception and piloted early work on dilution testing. DK: Developed the prototype dilution algorithm, contributed to the study conception and writing of manuscript. All authors read and approved the final manuscript.

\section{Acknowledgement}

The authors acknowledge the important support of Dr Asrat Genet Amnie and Dr. Zewdu Belew in the field sites. We also wish to acknowledge the contribution of the following individuals in executing the study in Abdurafi and Humera: Hayelom Zebrabruk, Million Wesine, Yisalem Amberbir, Fikadu Kassa, Hirut Tezera, Aderajew Kibret, Fasika Eyayu, Molla Dessie, Sahle Getachew, Biruk Assefa, Hafte Hadush, Kidane Geb. We thank the medical coordination team of Willemieke van den Broek, Endashaw Mengistu and Fareed Ahmed who provided much needed support for the study and Daniel Orozco and Joannie Roy who were involved in the implementation of the study.

\section{Author details \\ ${ }^{1}$ Médecins Sans Frontières, Amsterdam, The Netherlands. ${ }^{2}$ Médecins Sans Frontières, London, UK. ${ }^{3}$ Ethiopian Health and Nutrition Research Institute, Addis Ababa, Ethiopia. ${ }^{4}$ London School of Hygiene and Tropical Medicine, London, UK.}

Received: 2 January 2015 Accepted: 4 May 2015

Published online: 14 May 2015

\section{References}

1. World Health Organization. Service delivery approaches to HIV testing and Counselling (HTC). Geneva: WHO; 2012.
2. Gray RH, Makumbi F, Serwadda D, Lutalo T, Nalugoda F, Opendi P, et al. Limitations of rapid HIV-1 tests during screening for trials in Uganda: diagnostic test accuracy study. BMJ. 2007;335(7612):188.

3. Klarkowski DB, Wazome JM, Lokuge KM, Shanks L, Mills CF, O'Brien DP. The evaluation of a rapid in situ HIV confirmation test in a programme with a high failure rate of the WHO HIV two-test diagnostic algorithm. PLoS One. 2009;4(2):e4351.

4. Galiwango RM, Musoke R, Lubyayi L, Ssekubugu R, Kalibbala S, Ssekweyama $\checkmark$, et al. Evaluation of current rapid HIV test algorithms in Rakai, Uganda. J Virol Methods. 2013;192:25-7.

5. Aghokeng AF, Mpoudi-Ngole E, Dimodi H, Atem-Tambe A, Tongo M, Butel $\mathrm{C}$, et al. Inaccurate diagnosis of HIV-1 Group M and $\mathrm{O}$ is a key challenge for ongoing universal access to antiretroviral treatment and HIV prevention in Cameroon. PLoS One. 2009;4(11), e7702. doi:10.1371/journal.pone.0007702.

6. Klarkowski D, O'Brien DP, Shanks L, Singh KP. Causes of false positive HIV rapid diagnostic test results. Expert Rev Anti Infect Ther. 2013;12(1):49-62.

7. Clinical and Laboratory Standards Institute (CLSI). Criteria for Laboratory Test-ing and Diagnosis of Human Immunodeficiency Virus Infection; Approved Guideline. CLSI document M53-A (ISBN 1-56238-757-X [Print]; ISBN 1-56238-758-8 [Electronic]); 2011.

8. Centers for Disease Control and Prevention and Association of Public Health Laboratories. Laboratory Testing for the Diagnosis of HIV Infection: Updated Recommendations. Available at http://stacks.cdc.gov/view/cdc/23447. Published June 27, 2014. Accessed [August 14, 2014].

9. Shanks L, Siddiqui MR, Kliescikova J, Pearce N, Ariti C, Muluneh L, et al. Evaluation of HIV testing algorithms in Ethiopia: the role of the tie-breaker algorithm and weakly reacting test lines in contributing to a high rate of false positive HIV diagnoses. BMC Infect Dis. 2015;15:39. doi:10.1186/s12879-015-0769-3.

10. Shanks L, Klarkowski D, O'Brien DP. False positive HIV diagnoses in resource limited settings: operational lessons learned for HIV programmes. PLoS One. 2013;8:e59906. doi:10.1371/journal.pone.0059906.

11. Department of Health and Human Services/Centre for Disease Control and Prevention/African Regional Office of the World Health Organization. Guidelines for Appropriate Evaluations of HIV Testing Technologies in Africa. 2002.

12. Sandler SG, Dodd RY, Fang CT. Diagnostic tests for HIV infection: serology. In: De Vita VT, Hellman S, Rosenberg SA, editors. AIDS: etiology, treatment, and prevention. 2nd ed. Philadelphia: J.B. Lippincott; 1988. p. 121-6.

13. Pandori MW, Westheimer E, Gay C, Moss N, Fu J, Hightow-Weidman LB, et al. The Multispot rapid HIV-1/HIV-2 differentiation assay is comparable with the Western blot and an immunofluorescence assay at confirming HIV infection in a prospective study in three regions of theUnited States. J Clin Virol. 2013;58 Suppl 1:e92-6.

14. Malloch L, Kadivara K, Putzb J, Levett PN, Tang J, Hatchette TF, et al. Comparative evaluation of the Bio-Rad Geenius HIV-1/2 ConfirmatoryAssay and the Bio-Rad Multispot HIV-1/2 Rapid Test as an alternative differentiation assay for CLSI M53 algorithm-I. J Clin Virol. 2013;58S:e85-91.

15. World Health Organization Technical Working Group on HIV Incidence Assays. When and how to use assays for recent infection to estimate HIV incidence at a population level. Geneva: WHO; 2011.

16. Constantine NT, Sill AM, Jack N, Kreisel K, Edwards J, Cafarella T, et al. Improved classification of recent HIV-1 infection by employing a two-stage sensitive/less-sensitive test strategy. J Acquir Immune Defic Syndr. 2003;32:94-103.

17. Soroka SD, Granade TC, Candal D, Parekh BS. Modification of rapid human immunodeficiency virus (HIV) antibody assay protocols for detecting recent HIV seroconversion. Clin Diagn Lab Immunol. 2005;12:918-21.

18. Girardi SB, Egydio de Carvalho Barretob AM, Barretob CC, Proietti AB, Farias de Carvalhof SM, Loureiro P, et al. Evaluation of rapid tests for human immunodeficiency virus as a tool to detect recent seroconversion. Braz $J$ Infect Dis. 2012;16(5):452-6.

19. Kshatriya R, Cachafeiro AA, Kerr RJS, Nelson JAE, Fiscus SA. Comparison of two rapid Human Immunodeficiency Virus (HIV) assays, determine ${ }^{T M}$ HIV-1/2 and OraQuick advance rapid HIV-1/2, for detection of recent HIV Seroconversion. J Clin Microbiol. 2008;46(10):3482-3.

20. Bouillon $M$, Aubin E, Roberge $C$, Bazin R, Lemieux R. Reduced frequency of blood donors with false-positive HIV-1 and -2 antibody EIA reactivity after elution of low-affinity nonspecific natural antibodies. Transfusion. 2002;42(8):1046-52. 
21. Urwijitaroon Y, Barusrux S, Romphruk A, Puapairoj C, Thongkrajai P. Anti-HIV antibody titer: an alternative supplementary test for diagnosis of HIV-1 infection. Asian Pac J Allergy Immunol. 1997;15:193-8.

22. Duedu KO, Hayford AA, Sagoe KW. Misclassification of recent HIV-1 seroconversion in sub-Saharan Africa using the sensitive/less sensitive technique. Virol J. 2011;8:176.

23. Hahn JA, Wanyenze RK. Potential for false positive HIV test results with the serial rapid HIV testing algorithm. BMC Res Notes. 2012;5:154.

24. Boeras D, Luisi N, Karita E, McKinney S, Sharkey T, Keeling M, et al. Indeterminate and discrepant rapid HIV test results in couples' HIV testing and counselling centres in Africa. J Int AIDS Soc. 2011;14:18.

\section{Submit your next manuscript to BioMed Central} and take full advantage of:

- Convenient online submission

- Thorough peer review

- No space constraints or color figure charges

- Immediate publication on acceptance

- Inclusion in PubMed, CAS, Scopus and Google Scholar

- Research which is freely available for redistribution 